$\begin{array}{cl}\text { PRAMANA } & \text { (C) Indian Academy of Sciences } \\ \begin{array}{c}\text { journal of } \\ \text { physics }\end{array} & \begin{array}{l}\text { Vol. } 67, \text { No. } 5 \\ \text { November } 2006 \\ \text { pp. } 773-782\end{array}\end{array}$

\title{
New physics hints in $B$ decays and collider outlook
}

\author{
GEORGE W S HOU \\ Department of Physics, National Taiwan University, Taipei, Taiwan 10617, \\ Republic of China \\ E-mail: wshou@phys.ntu.edu.tw
}

\begin{abstract}
There are currently two hints for new physics involving $\mathrm{CP}$ violation in $b \rightarrow s$ transitions: $\Delta \mathcal{S} \equiv \mathcal{S}_{f}-\mathcal{S}_{J / \psi K} \neq 0$, and difference in direct CP asymmetry $\Delta \mathcal{A}_{K \pi} \equiv$ $\mathcal{A}_{K^{+} \pi^{0}}-\mathcal{A}_{K^{+} \pi^{-}} \neq 0$. We explore the two scenarios with a large and unique new $\mathrm{CP}$ phase in $b \leftrightarrow s$ transitions. Motivated by $\Delta S \neq 0$, we update on the right-handed strangebeauty squark $\widetilde{s b}_{1 \mathrm{R}}$ at $\mathrm{TeV}$ scale. Motivated by $\Delta \mathcal{A}_{K \pi} \neq 0$, we explore sequential fourth generation $t^{\prime}$ and $b^{\prime}$ quarks. Both scenarios can survive constraints such as SM level $b \rightarrow s \gamma$, sll and $B_{\mathrm{s}}$ mixing, and predict sizable $\mathrm{CP}$ violation in $B_{\mathrm{s}}$ mixing. The fourth generation picture predicts sizable $K_{\mathrm{L}} \rightarrow \pi^{0} \nu \nu$. Direct search for $\widetilde{s b}_{\mathrm{R}}, b^{\prime}$ and $t^{\prime}$ at hadronic colliders, such as Tevatron Run II and LHC, can complement further CP violation studies at these machines, as well as at the future Super $B$ factory.
\end{abstract}

Keywords. $B$ physics; flavor; CP violation; beyond Standard Model; rare decays; supersymmetry; fourth generation; collider.

PACS Nos 11.30.Er; 11.30.Hv; 12.60.Cn; 12.60.-i; 12.60.Jv; 13.25.Hw

\section{Introduction}

As we approach the era of 'flavor physics at/with the LHC' [1], the subject of New Physics (NP) in $B$ decays and its relation to collider physics is vast and pressing. However, $\mathrm{CP}$ violation (CPV) in $b \leftrightarrow s$ transitions is the current frontier for New Physics search, and we do have two hints from the $B$ factories. The first is the difference in mixing-dependent $\mathrm{CPV}$ (TCPV) as measured in penguin-dominated $b \rightarrow s \bar{q} q$ processes vs. tree-dominated $b \rightarrow s \bar{c} c$ processes. One expects no CPV in $B^{0} \rightarrow J / \psi K^{0}$ decay, hence, by measuring TCPV in this process, one expects to measure the CPV phase of $V_{t d}^{* 2}$ in $B^{0}-\bar{B}^{0}$ mixing. For the penguin-dominated $b \rightarrow s$ process such as $B^{0} \rightarrow \phi K^{0}$, within 3-generation Standard Model (SM3) one expects $S_{\phi K} \cong S_{J / \psi K}$ because there is practically no $\mathrm{CPV}$ phase in $V_{t s}$ that enters the penguin loop. The current [2] naive average over $b \rightarrow s \bar{q} q$ gives $S_{s \bar{q} q}=0.50 \pm 0.06$, compared with $S_{s \bar{c} c}=0.69 \pm 0.06$. This gives

$$
\Delta S \equiv S_{s \bar{q} q}-S_{s \bar{c} c} \simeq-0.19,
$$

which is $2.6 \sigma$ from zero, while theoretical expectation is $\Delta S>0[3,4]$ in SM3. The possible discrepancy emerged since 2003 and is rather widely discussed. It is 


\section{George W S Hou}

popular to involve strange-beauty squark mixings in the right-handed (RH) sector that enter penguin loops.

Less noticed is the difference in direct CPV (DCPV) asymmetries in $B^{0} \rightarrow K^{+} \pi^{-}$ and $B^{+} \rightarrow K^{+} \pi^{0}$ modes. DCPV is now firmly established [2] in $B$ decays, namely $\mathcal{A}_{K^{+} \pi^{-}}=-0.108 \pm 0.017$. However, for $B^{ \pm}$decay, $\mathcal{A}_{K^{+} \pi^{0}}=0.04 \pm 0.04$ is consistent with zero. The difference

$$
\Delta \mathcal{A}_{K \pi} \equiv \mathcal{A}_{K^{+} \pi^{0}}-\mathcal{A}_{K^{+} \pi^{-}} \simeq 0.15
$$

is close to $4 \sigma$ away from zero. Naively one expects the two to be very similar, and indeed theoretical predictions made before the experimental measurement of $\mathcal{A}_{K^{+} \pi^{-}}$typically gave $\Delta \mathcal{A}_{K \pi} \approx 0$. The difference in amplitude between $B^{0} \rightarrow$ $K^{+} \pi^{-}$and $B^{+} \rightarrow K^{+} \pi^{0}$ can arise from color-suppressed $C$, or from electroweak penguin (EWP) $P_{\mathrm{EW}}$. To invoke enhanced $C$, one might effectively suppress the CPV phase in tree amplitude for $B^{+} \rightarrow K^{+} \pi^{0}$. But to call on $P_{\mathrm{EW}}$, one would then definitely need NP CPV phase.

Since both hints involve CPV in $b \leftrightarrow s$ transitions, the underlying NP could be rather specific to these transitions and relatively hidden from other processes that are already well-studied. We illustrate the possible NP with two scenarios. One involves a strongly mixed right-handed 'strange-beauty' squark, which we call the $\widetilde{s b}_{1 \mathrm{R}}$ (the $\widetilde{s b}_{2 \mathrm{R}}$ is presumably much heavier). Being a squark that carries both strange and beauty flavor, it can induce $b \rightarrow s$ transitions via strong gluino loops, and largely affects strong penguins $(\mathrm{P})$ such as $b \rightarrow s \bar{q} q$, or $B_{\mathrm{s}}$ mixing. The $\mathrm{RH}$ nature induces new RH operators. The second scenario is a sequential fourth generation. The extra $t^{\prime}$ quark in penguin loops does not induce any new operators, but it affects more effectively the EWP operators, as well as $B_{\mathrm{s}}$ mixing. The accompanying $b^{\prime}$ could affect $D$ mixing. Both scenarios are intriguing in that they introduce a unique NP CPV phase that affects mainly $b \rightarrow s$ transitions.

The essence of new physics in flavor sector is that it has to survive the numerous, often stringent, lower energy constraints. Having survived these constraints, and having accounted for the possible NP hints, it is then interesting only when specific predictions can be made, such as in $B_{\mathrm{s}}$ mixing and associated CPV. As the LHC era approaches, in addressing these NP scenarios, we keep in mind collider links. These could be the aforementioned $B_{\mathrm{s}}$ mixing and CPV, but more interesting is the direct search for the culprits, i.e. $\widetilde{s b}_{\mathrm{R}}$ and $b^{\prime}, t^{\prime}$. It is exciting to be able to directly probe the source of flavor and $\mathrm{CP}$ violation at or below the TeV scale. We look forward to some unravelling in the near future.

\section{2. $\mathcal{S}_{\phi K}$ and strange-beauty squark}

The Standard Model (SM) has no flavor dynamics in the right-handed ( $\mathrm{RH}$ ) fermion sector, and we have no knowledge of the lower left elements of the fermion mass matrix $M_{i j}$, where $i, j$ are family indices. However, if we assume

$$
M_{i j} M_{j i} \approx M_{i i} M_{j j}
$$

then all elements of $M$ can be inferred, in the basis where the upper right elements of $\hat{M}^{(u)}=M^{(u)} / m_{t}$ and $\hat{M}^{(d)}=M^{(d)} / m_{\mathrm{b}}$ are close to $V_{\mathrm{CKM}}$. Equation (3) is 
reminiscent of 'commuting charges', and indeed it can be realized by Abelian flavor symmetries (AFS) [5]. One then notes that

$$
\hat{M}_{31}^{(d)} \sim \lambda, \quad \hat{M}_{32}^{(d)} \sim 1
$$

are rather sizable in strength, with $\hat{M}_{32}^{(d)}$ close to maximum. When one introduces supersymmetry (SUSY), these large flavor changing couplings are carried over to $\mathrm{RH}$ squarks. As phase freedom has already been used in the quark fields, $\tilde{d}_{i \mathrm{R}^{-}}$ $d_{j \mathrm{R}}-\tilde{g}$ strong dynamics is induced. It was pointed out that, because of stringent low-energy FCNC constraints in kaon sector, one needs four 'texture zeros' [6]. To focus on $b \leftrightarrow s$ transitions, then, one decouples the first generation [7], and focus on the $\tilde{s}_{\mathrm{R}}-\tilde{b}_{\mathrm{R}}$ squark sector, which naturally impact on $b \leftrightarrow s$ transitions. An alternative motivation [8] for this could come from near maximal $\nu_{\mu}-\nu_{\tau}$ mixing, and relating to $\tilde{s}_{\mathrm{R}}-\tilde{b}_{\mathrm{R}}$ mixing through SUSY-GUTS.

Our working picture is that squarks are relatively degenerate at some common scale $\widetilde{m}$ around the SUSY scale, with some approximate AFS that is not too far above. The off-diagonal elements of the LL squark mass matrix is CKM suppressed, while the LR and RL matrices are $m_{q} / \tilde{m}$ suppressed [9]. Thus, we have, for $\tilde{s}_{\mathrm{R}}-\tilde{b}_{\mathrm{R}}$ squark sector, the $2 \times 2 \mathrm{RR}$ submatrix

$$
\left(\widetilde{M}_{d}^{2}\right)_{R R}^{(s b)}=\left[\begin{array}{cc}
\widetilde{m}_{22}^{2} & \widetilde{m}_{23}^{2} \mathrm{e}^{-i \sigma} \\
\widetilde{m}_{23}^{2} \mathrm{e}^{i \sigma} & \widetilde{m}_{33}^{2}
\end{array}\right]=R\left[\begin{array}{cc}
\widetilde{m}_{1}^{2} & 0 \\
0 & \widetilde{m}_{2}^{2}
\end{array}\right] R^{\dagger},
$$

where the elements $\tilde{m}_{i j}^{2}$ are approximately equal. The unique new CP phase $\sigma$ is absorbed by the diagonalization matrix

$$
R \equiv\left[\begin{array}{cc}
\cos \theta & \sin \theta \\
-\sin \theta \mathrm{e}^{i \sigma} & \cos \theta \mathrm{e}^{i \sigma}
\end{array}\right]
$$

From approximate AFS, one expects near maximal mixing $\sin \theta \simeq 1 / \sqrt{2}$. Level splitting can in principle drive one state, the $\widetilde{s b}_{1 \mathrm{R}}$, light by large flavor mixing.

We stress that the new (and unique!) CPV phase $\sigma$ originates from right-handed $s-b$ quark sector, and is on the same footing as $\phi_{3} \equiv \arg V_{u b}^{*}$ of SM3. It is SUSY that allows this CPV phase to manifest itself. Perhaps a bit surprising is that, even with $\widetilde{s b}{ }_{1 \mathrm{R}}$ driven relatively light compared to $\tilde{m}>\mathrm{TeV}$, the scenario survives the normally stringent $b \rightarrow s \gamma$ constraint, even in the presence of large flavor violation in strong dynamics.

With the presence of right-handed dynamics, the number of operators are doubled. The operators $O_{1,2}^{\left({ }^{\prime}\right)}, O_{3-6}^{\left({ }^{\prime}\right)}, O_{7-10}^{\left({ }^{\prime}\right)}$ correspond to tree, strong penguin and EWP four-quark operators, and $O_{11,12}^{\left({ }^{\prime}\right)}$ are EM and strong dipole quark bilinear operators. The prime indicates changing from the LH operators in SM to RH operators induced by $\widetilde{s b} b_{1 \mathrm{R}}$. We remark that the impact of $\widetilde{s} b_{1 \mathrm{R}}$ is mostly on $O_{11}^{\prime}$ and $O_{12}^{\prime}$. In our numerical computations, we calculate the operator coefficients in the mass basis, the hadronic matrix elements are evaluated in naive factorization for simplicity, since we are evaluating NP CPV effects. 

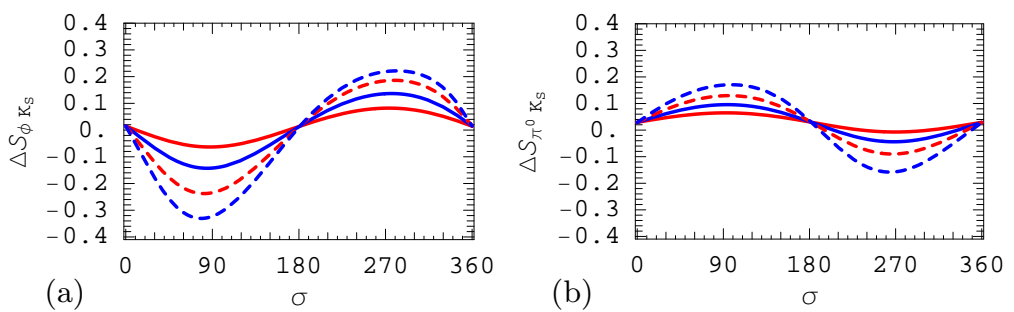

Figure 1. (a) $\Delta \mathcal{S}_{\phi K_{S}}$ and (b) $\Delta \mathcal{S}_{\pi^{0} K_{S}}$ vs. new CP phase $\sigma$ for $\tilde{m}=1.5$ $\mathrm{TeV}$. Solid curves are for $m_{\widetilde{s} b_{1}}=1100 \mathrm{GeV}$ and $750 \mathrm{GeV}$ with $m_{\tilde{g}}$ fixed at $950 \mathrm{GeV}$. Dashed curves are for $m_{\tilde{g}}=900 \mathrm{GeV}$ and $750 \mathrm{GeV}$ with $m_{\tilde{s} b_{1}}$ fixed at $500 \mathrm{GeV}$. Lighter combinations cause steeper curves.
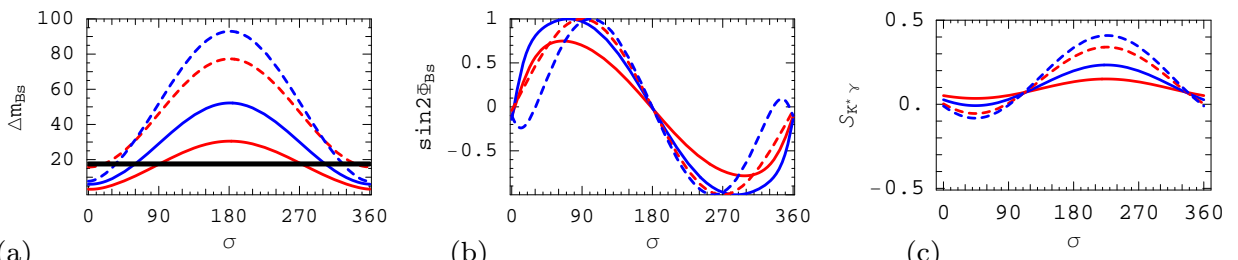

Figure 2. (a) $\Delta m_{B_{\mathrm{s}}}$, (b) $\sin 2 \Phi_{B_{\mathrm{s}}}$ and (c) $S_{K_{\gamma}^{*}}$ vs. $\sigma$ for $\tilde{m}=1.5 \mathrm{TeV}$. Notation is the same as for figure 1. The horizontal line in (a) is the $2 \sigma$ range for $\Delta m_{B_{\mathrm{s}}}$ measured by $\mathrm{CDF}$.

In 2003, $S_{\phi K_{S}} \sim-1$ was reported. We had to push $m_{\tilde{s b}_{1 \mathrm{R}}}$ to as low as $200 \mathrm{GeV}$ (which involves fine-tuning, if $\tilde{m}$ is maintained at above TeV) and $m_{\tilde{g}}$ as low as $500 \mathrm{GeV}$, to make $S_{\phi K_{S}} \sim-0.2$ [10], compared with $S_{J / \psi K_{S}} \simeq 0.73$. To push the number down further, we relegated it to hadronic uncertainties. The extreme situation of 2003 has since faded, and the deviation of $\Delta S \sim-0.2$ of eq. (1) is relatively mild and only $2.6 \sigma$. The pressure for a low $m_{\widetilde{s b}_{1 \mathrm{R}}}$ is no more. Although individual $\Delta S_{f}$ fluctuates between modes, as well as between Belle and BaBar measurements, we shall take four possible scenarios [11]: (1) $\Delta S_{\phi K_{S}} \sim-0.2$, (2) $\Delta S_{\pi^{0} K_{S}} \sim-0.2,(3)$ both $\Delta S_{\phi K_{S}}, \Delta S_{\pi^{0} K_{S}} \sim-0.2$, (4) both $\Delta S_{\phi K_{S}}, \Delta S_{\pi^{0} K_{S}} \sim 0$ (i.e. SM-like).

Our results are given in figure 1 , for various $m_{\widetilde{s b}}$ and $m_{\tilde{g}}$ combinations. Scenario (1) can be realized if one takes $\Delta S_{\pi^{0} K_{S}} \sim 0$. One finds $\sigma \sim 90^{\circ} \pm 60^{\circ}$, i.e. around $90^{\circ}$. Scenario (2) can be realized if one instead interprets $\Delta S_{\phi K_{S}} \sim 0$. One then has $\sigma \sim 270^{\circ} \pm 60^{\circ}$, i.e. around $270^{\circ}$. For Scenario (3), because of the ani-correlation between $\Delta S_{\phi K_{S}}$ and $\Delta S_{\pi^{0} K_{S}}$, this scenario where both these measurements are interpreted as actually negative is disfavored. Scenario (4) corresponds to SM-like results of $\Delta S_{\phi K_{S}, \pi^{0} K_{S}} \sim 0$. This is currently tolerated by data, and does not necessarily rule out $\widetilde{s} b_{1 \mathrm{R}}$. From figure 1 we see that $\pm 30^{\circ}$ around 0 or $180^{\circ}$ are tolerated by data.

$B_{\mathrm{s}}$ mixing has just been measured by CDF to be $\Delta m_{B_{\mathrm{s}}}=17.31_{-0.18}^{+0.33} \pm 0.07 \mathrm{ps}^{-1}$ [12], which is consistent with SM, but is a bit on the lower side. Using the same parameters as figure 1 , we predict $\Delta m_{B_{\mathrm{s}}}$ and the associated CPV $\sin 2 \Phi_{B_{\mathrm{s}}}$ in figure 2. The parameter range $90^{\circ} \lesssim \sigma \lesssim 270^{\circ}$ is clearly ruled out. In fact, what is left is 
the intersection of Scenarios (1) and (2) with Scenario (4). For lower $m_{\widetilde{s b}_{1}}$, a lower and narrower range for $|\sigma|$ is selected. For $m_{\tilde{g}} \sim 1 \mathrm{TeV}$ and $m_{\widetilde{s b}_{1}} \sim 0.75-1 \mathrm{TeV}$, one prefers $\sigma \sim 60^{\circ}-90^{\circ}$. This means that $\Delta S_{\phi K_{S}}$ (or $\Delta S_{\pi^{0} K_{S}}$ ) is probably weaker than eq. (1). But from figure $2 \mathrm{~b}$ we see that a rather large range for $\sin 2 \Phi_{B_{\mathrm{s}}}$ is possible for both signs. TCPV in $B_{\mathrm{s}}$ system may well be sizable. If $\left|\sin 2 \Phi_{B_{\mathrm{s}}}\right|$ is of order 1, CDF must have some sensitivity. At smaller values, we will have to wait for LHCb to start data taking, which could occur by late 2007.

A further measurable is of interest: TCPV in $B^{0} \rightarrow K^{* 0} \gamma \rightarrow K_{S} \pi^{0} \gamma$ [10]. BaBar and Belle have demonstrated the feasibility of measuring such mixing-dependent CPV, as a by-product of ' $K_{S}$-tagging' that allowed the measurement of $S_{\pi^{0} K_{S}}$. The error bars are still large, but one can look forward to great improvements at the Super $B$ factory, which aims at upgrading to 50-100 times current luminosities. On the physics side, the process is interesting as it probes the interference between $\mathrm{LH}$ and RH helicity photons [13],

$$
\mathcal{S}_{K^{*} \gamma}=\frac{2 \operatorname{Im}\left[\mathrm{e}^{-i 2 \phi_{1}} C_{11}^{\gamma} C_{11}^{\prime \gamma}\right]}{\left|C_{11}^{\gamma}\right|^{2}+\left|C_{11}^{\prime \gamma}\right|^{2}}
$$

where $\phi_{1}$ is the SM phase in $V_{t d}$ that is already measured. Because SM is LH only, $C_{11}^{\prime} / C_{11} \sim m_{\mathrm{S}} / m_{\mathrm{b}}$ is suppressed, and the SM expectation is at a few per cent level. With RH dynamics provided by $\widetilde{s b}_{1 R}$, which brings up $C_{11}^{\prime}$, we see from figure $2 \mathrm{c}$ that large positive values of $20-40 \%$ is in principle possible [10]. This is a welcome news for the Super $B$ factory. Unfortunately, with the measurement of $\Delta m_{B_{\mathrm{s}}}$ by CDF, the parameter space is much constrained. We see that larger values of $\mathcal{S}_{K^{*} \gamma}$ for $\sigma$ between 180 and $270^{\circ}$ are ruled out. For $\sigma \sim 40^{\circ}-60^{\circ}, \mathcal{S}_{K^{*} \gamma}$ centers around zero, while for $\sigma \sim 300^{\circ}-320^{\circ}, \mathcal{S}_{K^{*} \gamma}$ is around 0.1. Compounded by a new, larger range [14] of order 0.1 found for SM, it will be hard to distinguish between SM and $\widetilde{s b} b_{1 \mathrm{R}}$ effects. Perhaps if $\widetilde{s b_{1 \mathrm{R}}}$ effects are clarified at the LHC, and with further refinement of SM predictions, one can again distinguish between SM and $\widetilde{s b}_{1 \mathrm{R}}$ effect, by the time Super $B$ factory is in operation.

\section{3. $\mathcal{A}_{K \pi^{\circ}}$ Puzzle: Electroweak penguin and the fourth generation}

Enhancement of color-suppressed $C$ has been covered by the previous talk [15]. Here we expound on NP in $P_{\mathrm{EW}}$ by adding a sequential fourth generation $t^{\prime}$ to the usual $t$ loop.

The fourth generation fell out of favor shortly after the $N_{\nu}=3$ measurement by LEP and SLC. Currently, electroweak precision tests strongly disfavor the existence of a fourth generation, although this has not stopped efforts for direct search. Furthermore, there are loopholes in the electroweak precision physics.

So why do we invoke the fourth generation? Because it impacts on EWP most naturally, and brings in a new CPV phase. Let us first formulate how the fourth generation should be properly incorporated. Denoting $\lambda_{i} \equiv V_{i s}^{*} V_{i b}$, 3-generation CKM unitarity gives $\lambda_{u}+\lambda_{c}+\lambda_{t}=0$, which becomes $\lambda_{t} \cong-\lambda_{c}$ when one realizes that $\left|\lambda_{u}\right|$ is very small. Adding $t^{\prime}$, one has 


\section{George W S Hou}

$$
\lambda_{t} \cong-\lambda_{c} \quad \Longrightarrow \quad \lambda_{t} \cong-\lambda_{c}-\lambda_{t^{\prime}} .
$$

This means that, to the loop-induced amplitudes $\Sigma_{3}^{12} \lambda_{c} C_{i}^{t} O_{i}$, where the superscript $t$ indicates the SM3 contribution, one simply adds $-\lambda_{t^{\prime}}\left(C_{i}^{t^{\prime}}-C_{i}^{t}\right) O_{i}$, where $\Delta C_{i} \equiv$ $C_{i}^{t^{\prime}}-C_{i}^{t}$ is the GIM-respecting new penguin correction from $t^{\prime}$. It is well-known [16] that the $t^{\prime}$ effect is largely in $\Delta C_{9}$ and $\Delta C_{7}$ because of large $m_{t^{\prime}}$ effect. All other loop effects of $t^{\prime}$ in strong and EM penguins are highly GIM suppressed by insensitivity to heavy internal quark masses. In short, SM4 impacts on EWP naturally because of nondecoupling, which is traced to the Yukawa coupling that grows with $m_{\left.t^{(}\right)}$. Note also that the extra CKM coefficient,

$$
\lambda_{t^{\prime}} \equiv V_{t^{\prime} s}^{*} V_{t b} \equiv r_{s b} \mathrm{e}^{i \phi_{s b}},
$$

is naturally complex, just as $V_{t d}^{*} V_{t b}$ is complex in SM3. Given that eq. (2) is a NP hint in $b \rightarrow s$ that could arise from NP phase in $P_{\mathrm{EW}}$, the fourth generation is a very natural candidate, and should be taken seriously.

The $\mathcal{A}_{K \pi^{0}}$ problem emerged in the context of the experimental measurement of DCPV in $B^{0} \rightarrow K^{+} \pi^{-}$mode, where $\mathcal{A}_{K \pi}$ is found to be of order $-11 \%$. This measurement has confronted factorization models. Specifically, it favors the PQCD factorization approach, which predicted the sign and size of $\mathcal{A}_{K \pi}$ beforehand [17]. On the other hand, the more popular QCDF (QCD factorization) predicted [18] wrong sign for $\mathcal{A}_{K \pi}$ and at much smaller absolute value than observed. QCDF can parametrize the effect, e.g. in the so-called 's4' scenario [19], but this involves hadronic parameters that are no longer calculable, and one loses predictive power. In this sense, PQCD fared better than QCDF in the test of $\mathcal{A}_{K \pi}$. However, PQCD also predicted $\mathcal{A}_{K \pi^{0}} \sim \mathcal{A}_{K \pi}$, which is in contrast with eq. (2).

Let us first see how $\mathcal{A}_{K \pi}$ emerges in PQCD. In simplified notation and dropping the charge of $K$ and $\pi, \mathcal{M}(K \pi)=P+P_{\mathrm{a}}+T$, up to nonfactorizable terms. The penguin annihilation term $P_{\mathrm{a}}=\left|P_{\mathrm{a}}\right| \mathrm{e}^{i \delta_{P_{\mathrm{a}}}}$, which carries a strong, calculable phase $\delta_{P_{\mathrm{a}}}$ was one of the major observations made by PQCD. The upshot is,

$$
\mathcal{M}(K \pi) \propto \mathrm{e}^{i \delta}+r \mathrm{e}^{i \phi_{3}},
$$

where $\delta$ is the $\mathrm{CP}$ invariant phase of $P+P_{\mathrm{a}}, r=|T / P|$, and $\phi_{3}$ comes from $V_{u b}^{*}$ in $T$. For $B^{+} \rightarrow K^{+} \pi^{0}$

$$
\sqrt{2} \mathcal{M}\left(K \pi^{0}\right)-\mathcal{M}(K \pi)=P_{\mathrm{EW}}+C,
$$

plus a suppressed tree annihilation term and non-factorizable terms. Given that $P_{\mathrm{EW}}+C$ are also subleading, one has $\mathcal{A}_{K \pi^{0}} \sim \mathcal{A}_{K \pi}$ at leading order (LO) in PQCD, which corresponds to next-to-LO (NLO) in QCDF.

The PQCD people have pursued NLO corrections [20], and found some success in enhancing $C$. Let us, however, take the LO PQCD framework and incorporate the fourth generation. It is then easy to see that $\mathcal{M}(K \pi)$ is insensitive to EWP at leading order, while $t^{\prime}$ effects are subdued for strong penguin $P$. Thus, $\mathcal{M}(K \pi)_{\mathrm{SM} 4} \simeq \mathcal{M}(K \pi)_{\mathrm{SM} 3}$, and eq. (10) still holds. In contrast, eq. (11) receives the full force of $t^{\prime}$ corrections $\propto \lambda_{t^{\prime}}\left(\Delta C_{9}-\Delta C_{7}\right)$, bringing in the NP phase in $\arg \lambda_{t^{\prime}}$ and strong $m_{t^{\prime}}$ dependence in $\Delta C_{9}-\Delta C_{7}$. Similar pattern holds in $K^{0} \pi^{+}$ vs. $K^{0} \pi^{0}$ modes. 


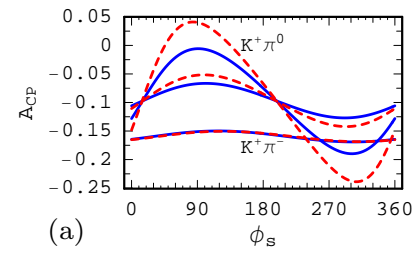

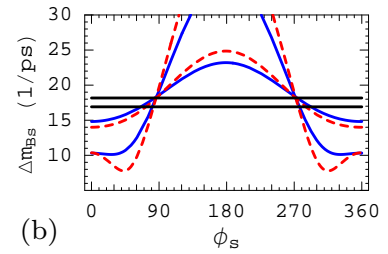

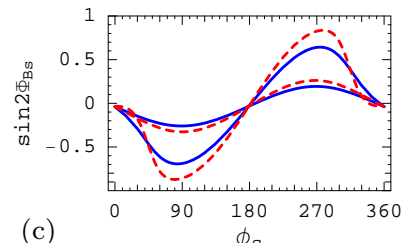

(c)

(b) $\Delta m_{B_{\mathrm{s}}}$ and (c) $\sin 2 \Phi_{B_{\mathrm{s}}}$ vs. $\phi_{\mathrm{s}} \equiv \arg V_{t^{\prime} s}^{*} V_{t^{\prime} b}$. The solid (dashed) curves are for $m_{t^{\prime}}=300$ (350) GeV and $r_{\mathrm{s}} \equiv\left|V_{t^{\prime} s}^{*} V_{t^{\prime} b}\right|=0.01,0.03$. The horizontal band in (b) is the $2 \sigma$ range for $\Delta m_{B_{\mathrm{s}}}$ measured by CDF.

We find that [21] eq. (2) can be attained, which in turn demands $\phi_{s b} \sim+90^{\circ}$, and the negative sign is ruled out. This is plotted in figure $3 \mathrm{a}$ vs. CP phase $\phi_{s b}$ for some parameter range. The needed effect is large, driving one to sizable $m_{t^{\prime}}$, and $r_{s b}=\left|V_{t^{\prime} s}^{*} V_{t^{\prime} b}\right|$ that is not much smaller than $V_{c s}^{*} V_{c b} \sim 0.04$.

Even before $\Delta m_{B_{\mathrm{s}}}$ measurement, one had to face stringent constraints from $b \rightarrow$ $s \ell^{+} \ell^{-}$and $B_{\mathrm{s}}$ mixing bound. The former is consistent with SM3, while the latter was a lower bound [22] not far from SM3 expectations. In fact, from $\mathcal{B}\left(b \rightarrow s \ell^{+} \ell^{-}\right)$ being consistent with SM3, one could already infer that, $\Delta m_{B_{\mathrm{s}}}$ in SM4 must be consistent with SM3 expectation as well. The PDG 2004 bound on $\Delta m_{B_{\mathrm{s}}}$, however, rules against too much destruction between $t^{\prime}$ and $t$ contributions, and combining with $b \rightarrow s \ell^{+} \ell^{-}, \phi_{s b} \sim \pm \pi / 2$ is preferred independent [21] of $\Delta \mathcal{A}_{K \pi} \neq 0$. That is, the preference is for the fourth generation effect to add in quadrature with SM3 top effect to minimize impact. This pushes down on the range for $m_{t^{\prime}}$ and $r_{s b}$, but some parameter space is allowed, e.g. $m_{t^{\prime}} \simeq 300 \mathrm{GeV}$ and $r_{s b} \sim 0.025$. Even so, the usually stringent $B \rightarrow X_{\mathrm{s}} \gamma$ constraint is easily satisfied, because $C_{11}$ is insensitive to heavy quark loop effects. DCPV in $b \rightarrow s \gamma$, however, turns out to be consistent with zero for the preferred parameter choice, and is considerably weaker than SM3 expectations. This dampens hope for measurement even at Super-B.

With the recent CDF measurement of $\Delta m_{B_{\mathrm{s}}}$, which is consistent with SM3, the implications are rather interesting. On one hand, to have large effect in $\Delta \mathcal{A}_{K \pi}$, $\phi_{s b} \sim+\pi / 2$ is preferred. On the other hand, since the CDF value for $\Delta m_{B_{\mathrm{s}}}$ is slightly on the low side, $\phi_{s b}<90^{\circ}$ is preferred and so the real part of $t^{\prime}$ effect is destructive against SM3. As plotted in figures $3 \mathrm{~b}$ and $3 \mathrm{c}$, we see that $\sin 2 \Phi_{B_{\mathrm{s}}} \sim$ -0.2 to -0.6 is predicted. It would be interesting to see if this prediction can be tested even at the Tevatron Run II.

Our further study [23] clarifies how CPV in $b \rightarrow s$ transitions could be large, but we never knew about it until the hints of eqs (1) and (2) emerged. The strength of $\mathrm{CPV}$ in $b \rightarrow s$ is illustrated by figure $4 \mathrm{a}$, a quadrangle that appears like a triangle. The corresponding SM3 triangle (with $V_{u s}^{*} V_{u b}$ exaggerated) is close to a line. Thus, impact on CPV in $b \rightarrow s$ from $t^{\prime}$ is large. In contrast, the quadrangle for $b \rightarrow d$, as illustrated in figure $4 \mathrm{~b}$, is barely distinguishable from the SM3 triangle. Thus, the impact on $b \rightarrow d$ transitions, including CPV measurement in $B \rightarrow J / \psi K_{S}$, is rather small. However, having large CPV phase in $V_{t^{\prime} s}^{*}$ with $\left|V_{t^{\prime}}\right|$ sizable, some fine tuning of $V_{t^{\prime} d}^{*}$ is necessary to give the correct $\varepsilon_{K}$ value. Together with saturating the $Z \rightarrow b \bar{b}$ constraint on $\left|V_{t^{\prime}}\right|$, we find that the $4 \times 4$ CKM matrix is more or less 

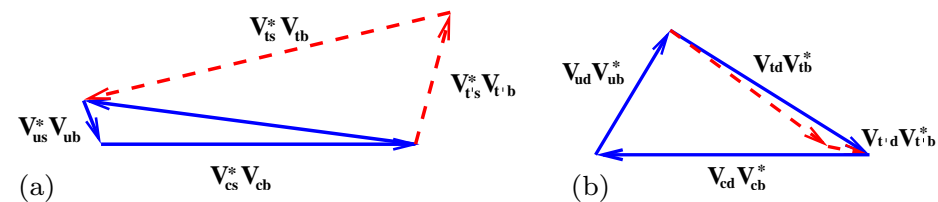

Figure 4. The four generation unitarity quadrangle for (a) $b \rightarrow s$ (with $V_{u s}^{*} V_{u b}$ exaggerated), (b) $b \rightarrow d$ processes. Actual scale for (b) should be $\sim 1 / 4$ in size. Solid lines represent the SM3 triangle.

fixed, with an interesting structure. As a by-product of the fix on $\varepsilon_{K}$ (and $\varepsilon_{K}^{\prime}$ as well), we find a large enhancement for the CPV decay $K_{\mathrm{L}} \rightarrow \pi^{0} \nu \nu$, which is close to saturating the Grossman-Nir bound [24] of $\sim 10^{-9}$. We also find that $D$ mixing, through finite $V_{c b^{\prime}}$ and $b^{\prime}$ loops, would not be too far below current bounds. These interesting phenomena can be probed in the near future.

It is interesting to note [25] that the fourth generation produces a downward dip for both $S_{\phi K}$ and $S_{\pi^{0} K}$. The effect is more prominent in LO PQCD. We have further checked that the effect is rather robust against hadronic uncertainties in QCDF, although the effect is somewhat weaker than in PQCD. Thus, the fourth generation could be also behind the $\Delta \mathcal{S}$ problem, generating consistent downward shift in various modes. For $S_{\eta^{\prime} K}$, however, our study with QCDF indicates that the NP effect gets diluted away by the large CP invariant correction to the amplitude that is needed to get the large rate. We believe this observation is generic for NP models.

Working at NLO in PQCD, it is shown that color-suppressed $C$ contribution to $B \rightarrow K \pi$ amplitude gets enhanced [15], but one may still need EWP since $\Delta \mathcal{S}$ is untouched within SM3. Work is in progress to combine fourth generation effect and NLO PQCD corrections, to see whether a better solution to both $\Delta \mathcal{S}$ and $\Delta \mathcal{A}_{K \pi}$ problems can be attained.

\section{Collider link: Direct search for $\widetilde{s b}_{1 \mathrm{R}}, b^{\prime}, t^{\prime}$}

One major contribution from colliders to $B$ physics would be the advent of $B_{\mathrm{s}}$ studies, with the recent measurement of $\Delta m_{B_{\mathrm{s}}}$ as harbinger. It would be interesting to see what the remaining Tevatron Run II program could do to probe TCPV in $B_{\mathrm{s}}$ mixing, i.e. $\sin 2 \Phi_{B_{\mathrm{s}}}$, which is expected to be $\sim-4 \%$ in SM3. As we have discussed, taking eq. (1) as hint, the strange-beauty $\widetilde{s b_{1 \mathrm{R}}}$ squark could give large $\sin 2 \Phi_{B_{\mathrm{s}}}$ of either sign. For the fourth generation, taking eq. (2) as hint, and with the possibility of accounting (at least partially) for eq. (1), $\sin 2 \Phi_{B_{\mathrm{s}}} \sim-0.2$ to -0.6 is predicted. Even if the first hint for $\sin 2 \Phi_{B_{\mathrm{s}}} \neq 0$ does not come from Tevatron Run II, we will learn rather soon once LHCb collects data!

Even more interesting is the direct search for these culprits of flavor and $\mathrm{CP}$ violation at hadronic colliders. An earlier study [26] showed that Tevatron Run II could discover (>10 events) a light $\widetilde{s b}_{1 \mathrm{R}}$ up to $300 \mathrm{GeV}$ with $2 \mathrm{fb}^{-1}$. As production is the same as the regular $\tilde{b}$ squark, the cross-section is not a problem. The key is to check the single and double $b$-tagged events against expected cross-section, as 
$\widetilde{s b} b_{1 \mathrm{R}} \rightarrow s, b$ (in association with the LSP, assumed to be the bino, or possibly the gravitino) with ratio $\cos ^{2} \theta, \sin ^{2} \theta$, where $\theta$ is the $\tilde{s}_{\mathrm{R}}-\tilde{b}_{\mathrm{R}}$ mixing angle.

Unfortunately, with the CDF measurement of $\Delta m_{B_{\mathrm{s}}}$ and the weakening of $\Delta \mathcal{S}$ problem, this low mass $\widetilde{s b}_{1 \mathrm{R}}$ is no longer viable, and $\widetilde{s} b_{1 \mathrm{R}}$ is out of reach for the Tevatron. It should be kept in mind, however, that if SUSY and the approximate AFS is above TeV scale, having $\widetilde{s b}_{1 \mathrm{R}}$ at $\mathrm{TeV}$ scale is in fact more natural. This is independent of having a hint in eq. (1) or not. That is, approximate AFS corresponds to a wide class of models, and the near maximal $s_{\mathrm{R}}-b_{\mathrm{R}}$ mixing is rooted in $V_{c b} \sim m_{\mathrm{s}} / m_{\mathrm{b}}$. Supersymmetrizing the approximate AFS is again rather general. So $\mathrm{TeV}$ scale is the natural frontier domain, hence having $\Delta \mathcal{S}$ not stronger than eq. (1) provides the natural setting. With LHC turn-on approaching, the TeV mass range can be readily explored.

The search for FCNC involving the top quark has been of interest since its discovery. Any measurement likely spells NP, since SM3 prediction is infinitesimal compared to what is realistically possible in the near future. We have checked [27] that $b^{\prime}$ can at best enhance $t \rightarrow c$ transitions to $10^{-6}$ order, still inaccessible at the LHC.

What is more interesting is the direct search for the $b^{\prime}$ and $t^{\prime}$ quarks themselves, and searching for their FCNC decays. It is important to realize that the LHC is the first machine where the existence (or not) of a sequential fourth generation can be studied directly, and once and for all. A recent study [27] shows that $t^{\prime} \rightarrow b W$ would by far dominate, hence $t^{\prime}$ search is just like searching for a heavier top quark. The phenomenology of $b^{\prime}$, however, is rather rich. For $m_{b^{\prime}}<m_{t}+M_{W}$ [28], $b^{\prime} \rightarrow t W^{*}$ is kinematically suppressed, and one may have $b^{\prime} \rightarrow c W, b Z, b H$ and $t W^{*}$ all at similar rate. One has the possibility of $c$-jets with $W, \mathrm{FCNC}$ decay dominance, and a possible bonus discovery of the Higgs boson. Above $t W$ threshold, one expects $b^{\prime} \rightarrow t W$ dominance, with the intriguing signature of $t \bar{t} W^{+} W^{-}$, or $4 W \mathrm{~s}$ plus two $b$-jets. Interestingly, the modes $b^{\prime} \rightarrow s Z, s H, s g$ may not be much lower than the $b^{\prime} \rightarrow b$ counterparts, and these modes could exhibit large direct CP violation. Unlike the situation in $B$ meson decays, where DCPV such as $\mathcal{A}_{K \pi}$ depends on hadronic phases that suffer from hadronic uncertainties, the CP invariant phases involved in $b^{\prime} \rightarrow s$ transitions are of short distance and calculable. Thus, the measurement of DCPV in $b^{\prime} \rightarrow s$ transitions (after the discovery of $b^{\prime}$ of course) could allow one to extract the fundamental CP violating phase. The search for $b^{\prime}$ should be pursued continuously at the Tevatron Run II, and absolutely at the LHC, to either open, or close, a chapter on fermion flavor.

\section{Summary}

Two hints from CP violation in $b \rightarrow s$ transitions, eqs (1) and (2), have emerged from $B$ factories, which could imply new physics. We have illustrated with two scenarios. One is a TeV scale 'strange-beauty' squark $\widetilde{s b_{1 \mathrm{R}}}$, the other is a sequential fourth generation with sizable $V_{t^{\prime}}$. Both bring a unique CPV phase into $b \rightarrow$ $s$ processes, and the recent measurement of $B_{\mathrm{s}}$ mixing by CDF provides a new, stringent constraint. Large $\sin 2 \Phi_{B_{\mathrm{s}}}$ in time-dependent $\mathrm{CP}$ violation in $B_{\mathrm{s}}$ system 


\section{George W $S$ Hou}

is predicted. We look forward to further tests, as well as direct search for $\widetilde{s b}_{1 \mathrm{R}}$ and $b^{\prime}, t^{\prime}$ at the LHC.

\section{Acknowledgement}

This work is supported in part by NSC 94-2112-M-002-035.

\section{References}

[1] See http://mlm.home.cern.ch/mlm/FlavLHC.html.

[2] Heavy Flavor Averaging Group (HFAG), http://www.slac.stanford.edu/xorg/hfag/

[3] M Beneke, Phys. Lett. B620, 143 (2005)

[4] H Y Cheng, C K Chua and A Soni, Phys. Rev. D72, 014006 (2005)

[5] Y Nir and N Seiberg, Phys. Lett. B309, 337 (1993)

M Leurer, Y Nir and N Seiberg, Nucl. Phys. B420, 468 (1994)

[6] C K Chua and W S Hou, Phys. Rev. Lett. 86, 2728 (2001)

[7] A Arhrib, C K Chua and W S Hou, Phys. Rev. D65, 017701 (2001)

[8] D Chang, A Masiero and H Murayama, Phys. Rev. D67, 075013 (2003)

[9] It turns out that, due to chiral enhancement, the $L R$ and $R L$ terms make numerical impact. This effect is included in actual computations

[10] C K Chua, W S Hou and M Nagashima, Phys. Rev. Lett. 92, 201803 (2004)

[11] W S Hou and M Nagashima, hep-ph/0602124

[12] CDF-Run II Collaboration: A Abulencia, Phys. Rev. Lett. 97, 062003 (2006)

[13] D Atwood, M Gronau and A Soni, Phys. Rev. Lett. 79, 185 (1997)

[14] B Grinstein, Y Grossman, Z Ligeti and D Pirjol, Phys. Rev. D71, 011504 (2005)

[15] H n Li, hep-ph/0605331, Pramana - J. Phys. 67(5), 755 (2006)

[16] W S Hou, R S Willey and A Soni, Phys. Rev. Lett. 58, 1608 (1987)

[17] Y Y Keum, H n Li and A I Sanda, Phys. Rev. D63, 054008 (2001)

[18] M Beneke, G Buchalla, M Neubert and C T Sachrajda, Nucl. Phys. B606, 245 (2001)

[19] M Beneke and M Neubert, Nucl. Phys. B675, 333 (2003)

[20] H n Li, S Mishima and A I Sanda, Phys. Rev. D72, 114005 (2005)

[21] W S Hou, M Nagashima and A Soddu, Phys. Rev. Lett. 95, 141601 (2005)

[22] Particle Data Group: S Eidelman et al, Phys. Lett. B592, 1 (2004)

[23] W S Hou, M Nagashima and A Soddu, Phys. Rev. D72, 115007 (2005)

[24] Y Grossman and Y Nir, Phys. Lett. B398, 163 (1997)

[25] W S Hou, M Nagashima, G Raz and A Soddu, J. High Energy Phys. 0609, 012 (2006)

[26] K Cheung and W S Hou, Phys. Rev. 70, 035009 (2004)

[27] A Arhrib and W S Hou, hep-ph/0602035, J. High Energy Phys. 0607, 009 (2006)

[28] W S Hou and R G Stuart, Phys. Rev. Lett. 62, 617 (1989); Nucl. Phys. B320, 277 (1989) 